\title{
Bidirectional 1.25-Gbps WDM-PON with Broadcasting Function Using A Fabry-Perot Laser Diode and RSOA
}

\author{
Thang T. Pham, Hyun-Seung Kim, Yong-Yuk Won, and Sang-Kook Han* \\ Department of Electrical \& Electronic Engineering, Yonsei University, Shinchon-dong, \\ Seodaemoon-gu, Seoul, Korea
}

(Received October 30, 2008 : revised December 3, 2008 : accepted December 4, 2008)

\begin{abstract}
A novel WDM-PON system delivering bidirectional 1.25-Gbps data and broadcasting data is proposed. A subcarrier signal modulates optical carriers of a Fabry-Perot-laser-diode based broadband light source to broadcast to all users. Reflective semiconductor optical amplifiers are used as modulators for the baseband data at both the optical line terminal and the remote optical network unit for a channel. Bit error rate and error vector magnitude were measured to demonstrate the proposed scheme.
\end{abstract}

Keywords : Broadcast, Fabry-Perot laser diode, Reflective semiconductor optical amplifier, Wavelengthdivision-multiplexed passive optical network.

OCIS codes : (060.2360) Fiber optics links and subsystems; (060.4252) Networks, broadcast; (060.4230) Multiplexing

\section{INTRODUCTION}

Wavelength division multiplexed passive optical networks (WDM-PON) have been considered as an attractive solution for an access network. It has highly efficient use of the outside fiber by providing point-to-point connection to multiple remote optical network units (ONU) through just a single feeder fiber. Furthermore, for each connection, it has many advantages such as large bandwidths, bit rate independency, protocol transparency, easy upgradeability and excellent security [1-10].

To meet the demands of users for the diversity of services, it is important for the WDM-PON to deliver many kinds of multimedia data such as voice service, internet and broadcasting data. However, in the WDMPON, virtual point-to-point connection based on an Arrayed Waveguide Grating (AWG) causes the wavelengthselectivity characteristic of ONUs [5, 6]. Consequently, it is difficult to simultaneously transmit both the broadcasting data and bidirectional baseband data to all ONUs with a low cost. To address this problem, several broadcasting methods in WDM-PON architectures have been proposed recently. The overlay approach utilizes a broadband light source (BLS) and the cyclic property of an

*Corresponding author: skhan@yonsei.ac.kr
AWG [5-7]. All of these schemes, however, are costly because they require a WDM device at each ONU to separate two wavelengths and another PD to detect the broadcasting data. Another approach, in which broadcasting data combined with baseband data directly modulate an optical source at OLT for each ONU, overcomes the drawback of the wavelength-selectivity characteristic [8]. However, because a broadcasting data source and a DFB are needed for a certain channel, it also makes the system expensive. A low cost BLS for both baseband data and broadcasting data using mutually injected Fabry-Perot laser diodes (FP-LD) has been proposed [9]. Nevertheless, the mutual injection locking structure is complicated and bandwidth for data transmission is limited due to noise peaks generated by the external cavity.

In this paper, we present a cost-effective, colorless and bidirectional local access WDM-PON architecture to transmit uplink/downlink baseband data and broadcasting data. A broadcasting subcarrier multiplexing (SCM) signal directly modulates a FP-LD based BLS. Gainsaturated reflective semiconductor optical amplifiers (RSOAs) are used as modulators for both the uplink and downlink baseband data for bidirectional transmission. We verified the validity of the proposed system by measuring the bit-error rate (BER) for the baseband data, 


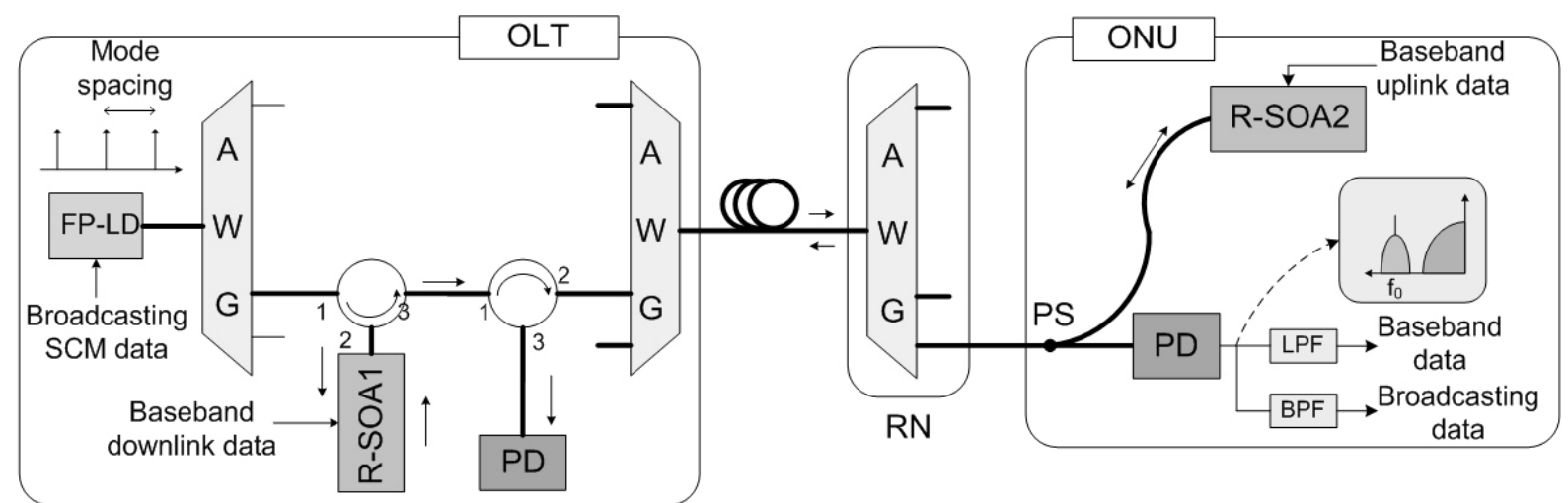

FIG. 1. Schematic of the proposed WDM-PON system.

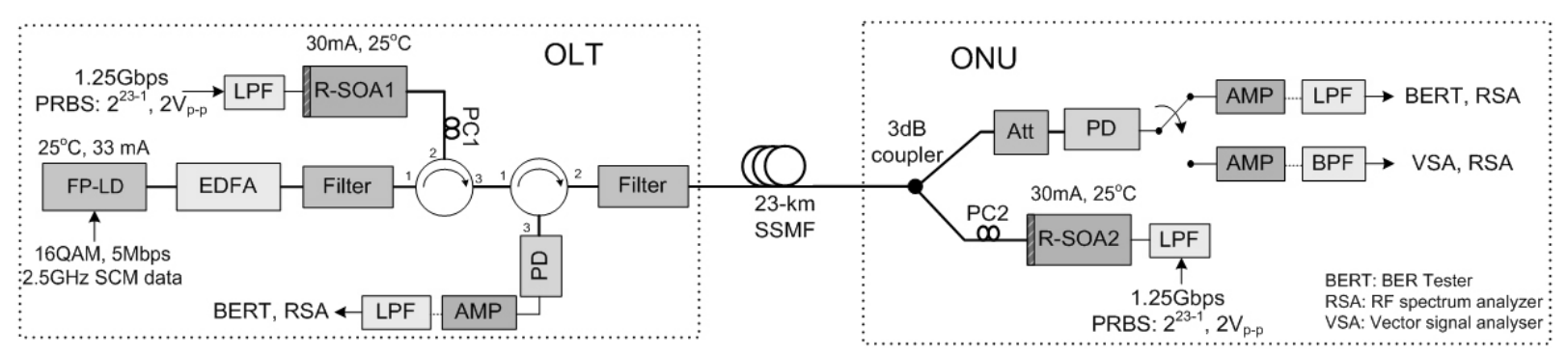

FIG. 2. Experimental setup for the proposed scheme.

error vector magnitude (EVM) for the broadcasting SCM data.

\section{PRINCIPLE OF OPERATION}

Fig. 1 shows the schematic of the proposed bidirectional WDM-PON system supporting broadcasting function. At the optical line terminal (OLT), a FP-LD is used as a multi-wavelength light source for many WDM channels. The broadcasting SCM signals directly modulate optical carriers at the FP-LD. Each mode of the FP$\mathrm{LD}$ is separated by an AWG and enters a gain-saturated RSOA (RSOA1). At this RSOA, first of all, mode partition noise (MPN) generated due to spectrally slicing the FP-LD optical output is reduced by high pass filter (HPF) effect of the gain-saturated RSOA $[10,11]$ and then the optical carrier is modulated by 1.25 -Gbps downlink data. After the RSOAs, the optical signals of all the channels are recombined by using another AWG and transmitted to the AWG-based remote node (RN). At the RN, the optical signal of each channel is separated again and transmitted to a certain ONU. The channel spacing of all the AWGs is equal to the mode spacing of FP-LD. At the ONU, this optical signal is divided into two paths by using a power splitter (PS). One path is detected by a photodetector (PD) to recover both the baseband and broadcasting data; the other path enters another RSOA (RSOA2). In gain-saturation condition of the RSOA2, the downlink optical carrier is remodulated by 1.25 -Gbps uplink baseband data and then retransmitted to the OLT, detected by another PD.

\section{EXPERIMENT AND RESULTS}

The experimental setup for the proposed scheme is illustrated in Fig. 2. The FP-LD has a mode spacing of $1.08 \mathrm{~nm}$ and main spectral gain region of from 1530 to $1550 \mathrm{~nm}$. Its operating temperature and biased current were $25^{\circ} \mathrm{C}$ and $33 \mathrm{~mA}$, respectively. A part of its optical spectrum is shown is Fig. 3(a). The SCM signal with 16-QAM modulation format, 5 Msps at $2.5-\mathrm{GHz}$ carrier frequency and $10-\mathrm{dBm}$ power directly modulated the optical carriers of the FP-LD. The optical signal from the FP-LD was amplified to reach a high power level before separated and injected into RSOAs. Instead of the AWGs, two tunable optical filters (TOF) were used to separate each channel. 1.25-Gbps NRZ data with 2- $\mathrm{V}_{\mathrm{p}-\mathrm{p}}$ modulated the optical carrier at the RSOAs for both the uplink and the downlink. To avoid interference between the baseband and the broadcasting signals, a low pass filter (LPF) with cut-off frequency of $1.2 \mathrm{GHz}$ was used to remove the high frequency components of the baseband signal before modulating the optical carrier at the RSOA. Optical transmission length was $23 \mathrm{~km}$. The optical signal of each downlink channel was divided into two 


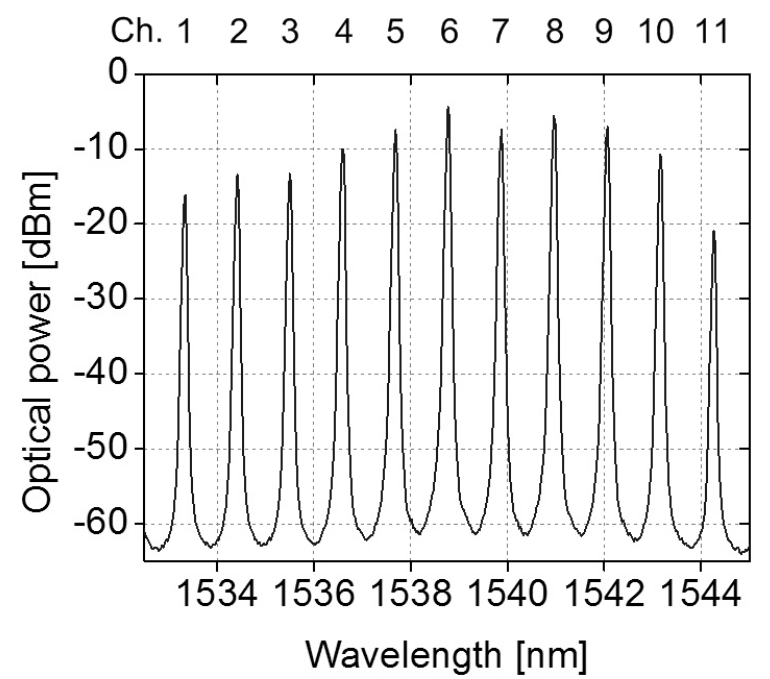

(a)

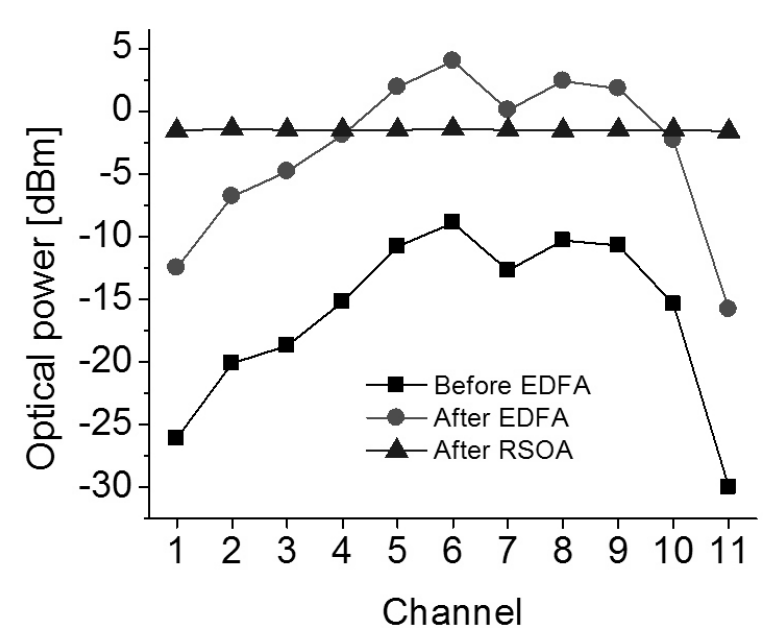

(b)

FIG. 3. a) Optical spectrum of FP-LD, b) optical power of channels

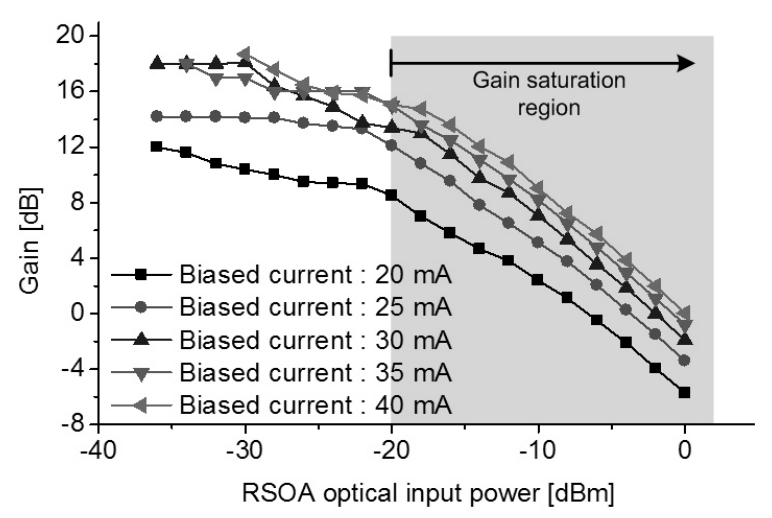

(a)

FIG. 4. a) Gain curves of RSOA, b) RF spectra.

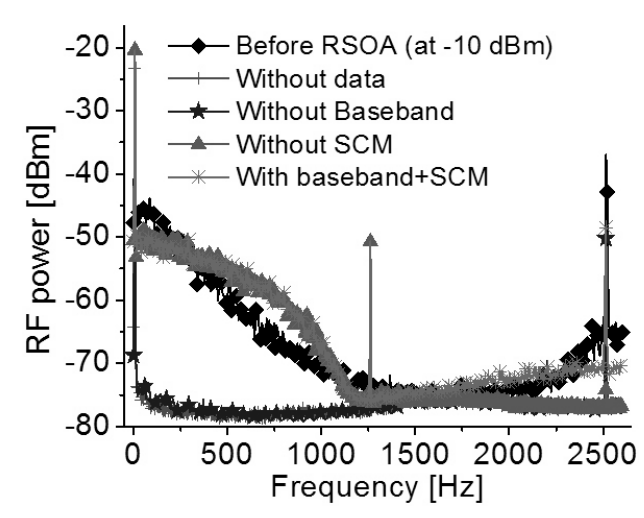

(b) paths by using a 3-dB coupler. The optical power of each path was about $-12 \mathrm{dBm}$ for all the channels. After the PD, the recovered broadcasting signal and the baseband signals were amplified using low noise amplifiers (LNA), filtered out and measured the performance. The RSOAs used in this experiment had the same specification. The gain curve of RSOA1 was measured with a tunable laser source (TLS) and illustrated in Fig. 4(a). As shown in this figure, when the injected power was over $-20 \mathrm{dBm}$, it operated under the gain saturation region. The temperature and biased current of the RSOAs were $25^{\circ} \mathrm{C}$ and $30 \mathrm{~mA}$, respectively.

For performance measurement, up to 11 channels were selected which were numbered from 1 to 11 as presented in Fig. 3(a). Most of the optical powers of all the channels from the FP-LD were small, which did not satisfy the requirement of the injected optical power of RSOA1 to work under the gain-saturation condition $(-20 \mathrm{dBm})$. With the optical gain from the
EDFA, the injected optical powers were satisfactory. In this condition, the output optical power of RSOA is about $-1.5 \mathrm{dBm}$ regardless of the different injected optical powers of all the channels as illustrated in Fig. 3(b). The spectra of the detected RF signal of channel 9 are described in Fig. 4(b) including before RSOA1, with/ without the data. As shown in this figure, the spectrally-sliced optical carrier from the FP-LD contained the MPN at low frequency region. Moreover, because of the square-law detection of the PD, MPN also appeared in the frequency region of the SCM signal $(2.5-\mathrm{GHz}$ band). After injection to the gain-saturated RSOA (RSOA1), MPN was reduced perfectly at both the low frequency band and the $2.5-\mathrm{GHz}$ band. In this proposed scheme, the SCM data and the baseband data modulate the optical carrier in two consecutive steps. As a result, the baseband data also embedded in the subcarrier. It would make the performance of the broadcasting data degraded. Fig. 5(a) shows the EVM curve of the SCM signal at $2.5-\mathrm{GHz}$ carrier at back to back (BtoB) 


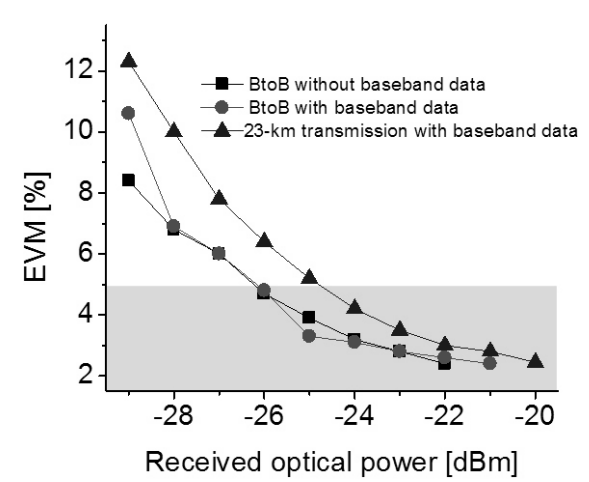

(a)

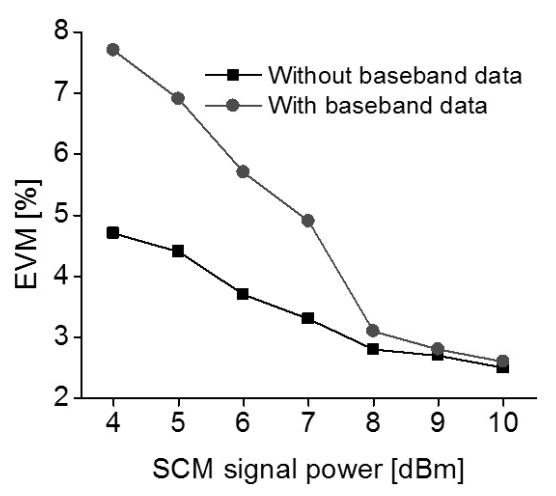

(b)

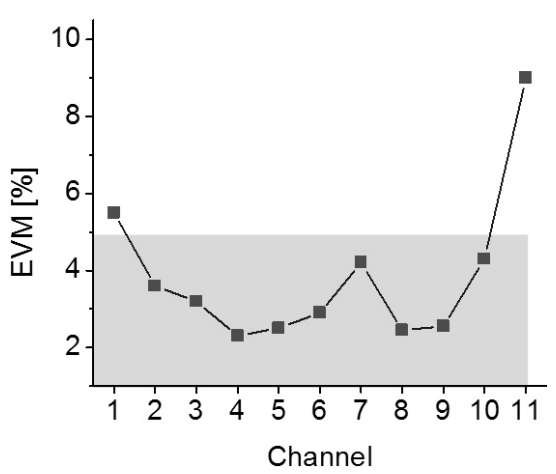

(c)

FIG. 5. Broadcasting signal performance: a) EVM curve of SCM signal, b) EVM with different RF power, c) EVM of different channels after transmission.

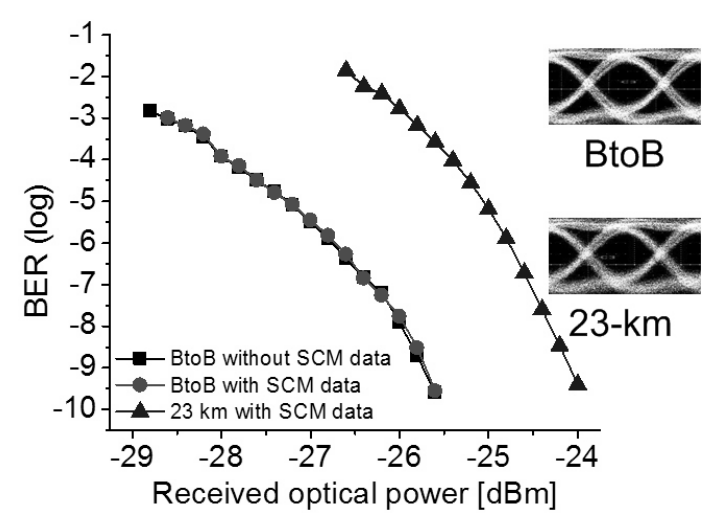

(a)

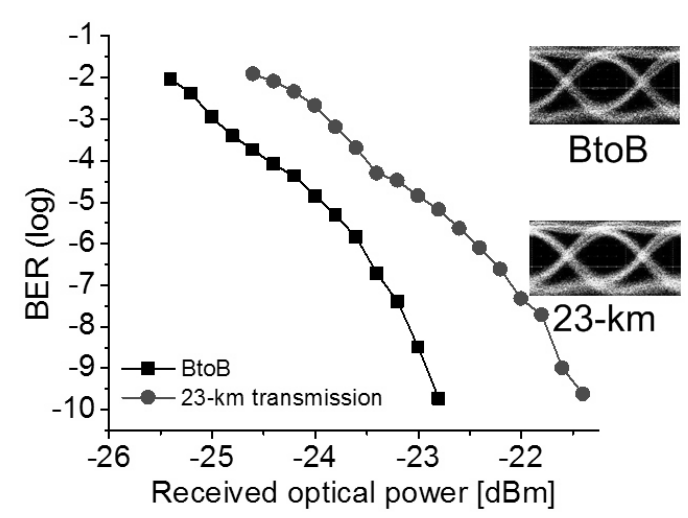

(b)

FIG. 6. Transmission performance of a) downlink baseband data, b) uplink baseband data.

with/without the baseband data and after 23-km transmission. The EVM values of about $2.5 \%$ were achieved at $-21 \mathrm{dBm}$ at BtoB and it was about 1-dB power penalty to achieve the same EVM after $23-\mathrm{km}$ transmission. The performance of SCM data was stable regardless of the existence of the downlink baseband data. It means that the performance degradation was insignificant in this condition since the signal to noise ratio (SNR) of the SCM signal was still high. However, when the power of the SCM signal decreased, EVM increased. This influence on the EVM of the SCM signal is described in Fig. 5(b) in case of after 23-km transmission and at -20-dBm optical power by using an optical attenuator. We chose this optical power point because it is the minimum requirement of optical power to satisfy gainsaturation condition of the RSOA for the uplink. The EVM values of 11 channels were also measured after 23-km transmission at $-20-\mathrm{dBm}$ optical power and are illustrated in Fig. 5(c). It was able to achieve high performance with EVM $<5 \%$ for 9 channels, from channel 2 to channel 10. For channel 1 and 11, they had lower performance because as shown in Fig. 3, they had a low optical signal-to-noise ratio (OSNR) compared with the other channels.
As pointed out in other works by author [10, 11], with the implementation of the RSOAs as modulators at both the OLT and ONT for each channel, error-free transmission for both the uplink and downlink baseband data is achievable for many channels without any effect of the MPN. In this paper, only BER curves for both the downlink and uplink data of channel 9 were measured which are shown in Fig. 6(a) and 6(b). Error-free (BER $<10^{-9}$ ) transmission at BtoB was achieved at $-25.8 \mathrm{dBm}$ for the downlink and $-23 \mathrm{dBm}$ for the uplink. Due to dispersion and reflection noises, the performance after $23-\mathrm{km}$ transmission was about $1.5 \mathrm{~dB}$ worse than BtoB. There is no difference of the BER characteristics between cases of with and without the SCM signal. This is because the frequency of this signal is far away from the main lope of the baseband data and it is removed perfectly by using a LPF with the cut-off frequency of $1.2 \mathrm{GHz}$.

\section{CONCLUSION}

We have proposed and demonstrated a simple and cost-effective WDM-PON architecture to deliver bidirec- 
tional baseband data and broadcasting data. Just only one FP-LD based BLS is required for many ONUs. A SCM signal at $2.5 \mathrm{GHz}$ is broadcasted to the ONUs by directly modulating this BLS. Gain-saturated RSOAs are used as optical modulator at both OLT and ONU for a channel to realize a colorless system. We achieved error-free transmission for the uplink/downlink baseband data and EVM $<5 \%$ for the broadcasting data for many channels. With up to $8 \mathrm{~dB}$ power budget for both the uplink and downlink, the transmission distance of the proposed WDM-PON system can be expanded while the performance is maintained.

\section{REFERENCES}

[1] Frigo, N.J.; Iannone, P.P.; Magill, P.D.; Darcie, T.E.; Downs, M.M.; Desai, B.N.; Koren, U.; Koch, T.L.; Dragone, C.; Presby, H.M., and Bodeep, G.E., "A wavelength-division multiplexed passive optical network with cost-shared components," IEEE J. Lightwave Tech., vol. 6, no.11, pp. 1365-1367, 1994.

[2] Soo-Jin Park, Chang-Hee Lee, Ki-Tae Jeong, Hyung-Jin Park, Jeong-Gyun Ahn, and Kil-Ho Song, "Fiber-to-thehome services based on wavelength-division-multiplexing passive optical network," IEEE J. Lightwave Tech., vol. 22, no. 11, pp. 2582-2591, 2004.

[3] Min-Hwan Kim, Sang-Mook Lee, Sil-Gu Mun, and ChangHee Lee, "A 240 km Reach DWDM-PON of 8-Gb/s Capacity using an Optical Amplifier,” J. Opt. Soc. Korea, vol. 11, no 3, pp. 93-96, 2007.

[4] Geun-Young Kim, Jin-Hee Kim, "Wavelength Division Multiplexing-Passive Optical Network Based FTTH Field
Trial Test," J. Opt. Soc. Korea, vol. 11, no 3, pp. 101107, 2007.

[5] Jung-Hyung Moon, Ki-Man Choi, and Chang-Hee Lee, "Overlay of broadcasting signal in a WDM-PON," in Proc. OFC 2006, Paper OThK8, 2006.

[6] Jinwoo Cho, Jaedon Kim, David Gutierrez, and Leonid G. Kazovsky, "Broadcast Transmission in WDM-PON using a Broadband," in Proc. OFC 2006, Paper OWS7, 2006.

[7] Jeung-Mo Kang, Sang-Hoon Lee,Hyuk-Choon Kwon, and Sang-Kook Han, "WDM-PON with Broadcasting Function Using Direct ASE Modulation of Reflective SOA,” in Proc. OFC 2006, Paper P.160, 2006.

[8] Tae-Young Kim and Sang-Kook Han, "Reflective SOABased Bidirectional WDM-PON Sharing Optical Source for Up/Downlink Data and Broadcasting Transmission," IEEE Photon. Technol. Lett., vol. 18, no. 22, pp. 23502352, 2006.

[9] Ho-Chul Ji, Ikuo Yamashita, and Ken-Ichi Kitayama, "Cost-effective colorless WDM-PON delivering up/downstream data and broadcasting services on a single wavelength using mutually injected Fabry-Perot laser diodes," Opt. Exp., vol. 16, no. 7, pp. 4520-4528, 2008.

[10] Thang T. Pham, Hyun-Seung Kim, Yong-Yuk Won, and Sang-Kook Han, "Bidirectional 1.25Gbps WDM RoF Wired/Wireless Optical Transmission using Multiple SSB Carriers in FP LD by Multiple Mode Injection Locking," in Proc. ECOC 2008, vol. 5, Paper P.6.21, 2008.

[11] Hyun-Seung Kim, Thang T. Pham, Yong-Yuk Won, and Sang-Kook Han, "1.25Gbps WDM-RoF Optical Transmission for Wired/Wireless Access Network using Multi Optical Carrier Suppression in FP LD," in Proc. Photonics in Switching 2008, Paper P-34, 2008. 